\title{
Store-operated Ca2+ entry: a key component of the insulin secretion machinery
}

\author{
Jessica Sabourin' ${ }^{1}$ and Florent Allagnat² \\ 1Signalisation et Physiopathologie Cardiovasculaire, Inserm UMR-S 1180-LabEx LERMIT, \\ Faculté de Pharmacie, Châtenay-Malabry France \\ 2Department of Vascular Surgery, Centre Hospitalier Universitaire Vaudois, Lausanne, Switzerland
}

Correspondence

should be addressed

to F Allagnat

Email

florent.allagnat@chuv.ch

\begin{abstract}
Normal plasma glucose level is ensured by the action of insulin, the major hypoglycemic hormone. Therefore, it is not surprising that insulin release from pancreatic $\beta$-cells of the islets of Langerhans is controlled by an array of balanced mechanisms in which glucose plays the leading role. Glucose triggers insulin secretion through the well-described pathway of ATP-driven closure of ATP-sensitive potassium channels $\left(\mathrm{K}_{\mathrm{ATP}}\right)$, depolarization of the plasma membrane, and opening of the voltage-dependent $\mathrm{Ca}^{2+}$ channels (VDCC). The subsequent rapid rise in cytoplasmic free $\mathrm{Ca}^{2+}$ concentration triggers insulin exocytosis. However, despite more than 40 years of investigation, certain aspects of the intracellular $\mathrm{Ca}^{2+}$ responses to glucose and secretagogues remain unexplained, suggesting the involvement of additional $\mathrm{Ca}^{2+}$ channels. Here, we discuss the emerging role of store-operated $\mathrm{Ca}^{2+}$ channels carried by Orai1 and transient receptor potential canonical 1 (TRPC1) proteins and regulated by the stromal interaction molecule 1 (STIM1) in the control of glucose-induced insulin secretion. The role of other voltageindependent cation channels formed by other members of the TRP channels family is also addressed.
\end{abstract}

\author{
Key Words \\ - store-operated $\mathrm{Ca}^{2+}$ entry \\ - TRPC1 \\ - STIM1 \\ - Orai1 \\ - insulin secretion \\ - pancreatic $\beta$-cells
}

\author{
Journal of Molecular \\ Endocrinology \\ (2016) 57, F35-F39
}

\section{Introduction}

Insulin secretion is driven by electrical activity and oscillations of intracellular $\mathrm{Ca}^{2+}$ concentration $\left(\left[\mathrm{Ca}^{2+}\right]_{\mathrm{i}}\right)$ in pancreatic $\beta$-cells. The main driver of insulin secretion is plasma glucose; glucose metabolism produces ATP, which closes ATP-sensitive potassium channels $\left(\mathrm{K}_{\mathrm{ATP}}\right)$ and cell depolarization, which, in turn, open voltagedependent $\mathrm{Ca}^{2+}$ channels (VDCC). The sudden rise in intracellular $\mathrm{Ca}^{2+}$ levels causes exocytosis of insulincontaining vesicles. In addition to $\mathrm{K}_{\mathrm{ATP}}$ channels and VDCC, a complex interplay between additional ion channels and intracellular $\mathrm{Ca}^{2+}$ stores produces unique patterns of oscillatory depolarization and $\left[\mathrm{Ca}^{2+}\right]_{\mathrm{i}}$ changes controlling insulin release (Gilon et al. 2014).

Since the concept of store-operated $\mathrm{Ca}^{2+}$ entry (SOCE) was first defined and characterized by Putney in the mid1980s (Putney 1986), evidence has accumulated indicating that SOCE exists almost in all cell types. However, while SOCE has been shown to play a critical role in agonistinduced responses in many non-excitable cell types, direct evidence for its role in excitable cells is generally lacking.

This review focuses on recent advances in the field of SOCE in insulin-secreting pancreatic $\beta$-cells.

Published by Bioscientifica Ltd 


\section{State of the art: current understanding of store-operated channels}

The ubiquitous SOCE, first described by Putney in 1986 (Putney 1986), is the prominent mechanism of $\mathrm{Ca}^{2+}$ entry in nearly all non-excitable cells. The activation of a variety of phospholipase C (PLC)-coupled receptors leads to the formation of two second messengers, diacylglycerol (DAG) and inositol-1,4,5-trisphosphate $\left(\mathrm{IP}_{3}\right)$. In turn, the $\mathrm{IP}_{3}$ receptors mobilizes $\mathrm{Ca}^{2+}$ from the endoplasmic reticulum (ER), resulting in depletion of $\mathrm{Ca}^{2+}$ stores, which triggers extracellular $\mathrm{Ca}^{2+}$ influx named SOCE. In addition to rapidly refilling the depleted ER stores, which is essential to maintain proper ER functions, SOCE also triggers downstream signaling events involved in a wide variety of cell functions such as proliferation, growth, migration, inflammation and apoptosis. In addition, SOCE has been proposed to contribute to short-term cellular responses such as $\mathrm{Ca}^{2+}$ oscillations, exocytosis and muscle contractility. Despite the growing acknowledgment of the biological importance of SOCE into well-established models of $\mathrm{Ca}^{2+}$ homeostasis, the molecular mechanisms responsible for sensing $\mathrm{ER} \mathrm{Ca}^{2+}$ depletion and triggering extracellular $\mathrm{Ca}^{2+}$ influx were the subject of intense debate since 1986, until the identification of stromal interaction molecule 1 (STIM1) in 2005. The STIM proteins are ER transmembrane proteins with a luminal C-terminal domain working as a $\mathrm{Ca}^{2+}$ sensor. Emptying of the ER $\mathrm{Ca}^{2+}$ stores sensed by this domain induces the rapid oligomerization of STIM1 dimers and translocation to $\mathrm{ER} /$ plasmalemmal junctional regions, where they physically interact with store-operated channels (SOCs) to induce SOCE. Despite high homology, the two isoforms, STIM1 and STIM2, have subtly distinct functions. STIM1 seems to be the main SOC-activating isoform, while STIM2, although its function is not completely elucidated, is a more sensitive sensor of ER luminal $\mathrm{Ca}^{2+}$ (reviewed in Prakriya 2013).

Early studies supported that the transient receptor potential canonical (TRPC) channels family formed SOCs. The seven members of this family (TRPC1-C7) assemble as homo- or heterotetramers to form cation-permeable channels with modest $\mathrm{Ca}^{2+}$ selectivity. These channels are activated in response to receptor-mediated PLC activation via store-dependent and store-independent mechanisms. They typically generate relatively linear current-voltage relationships called ISOC with a reversal potential near $0 \mathrm{mV}$. However, the involvement of TRPC channels in the SOCs formation remains a highly contentious and unresolved issue (reviewed in Cheng et al. 2013).
In addition to TRPC proteins, the discovery in 2005 of the interaction between STIM1 and Orai1 proteins has created a whole new field of SOCE research. Orai1, which was first identified in mast and $\mathrm{T}$ cells via RNAi-mediated knockdown strategy, recapitulates the archetypical $\mathrm{Ca}^{2+}$ release-activated $\mathrm{Ca}^{2+}$ (CRAC) current ( $I_{\text {CRAC }}$ ) (Prakriya 2013). This $I_{\text {CRAC }}$ current is characterized by its responsiveness to store depletion, its small singlechannel and whole-cell current densities, and its extreme selectivity for $\mathrm{Ca}^{2+}$ under physiological conditions (reversal potential near $+50 \mathrm{mV}$ ). There are three human Orai proteins (Orai1-3), but little is known about Orai2 and -3 . The interaction between the cytoplasmic region of STIM1 (Orai-activating small fragment residues) and the C-terminal cytosolic segment of Orai1 is mandatory for the $I_{\mathrm{CRAC}}$ current (Prakriya 2013).

Several recent reports suggest that TRPC proteins, especially TRPC1 and -C4, interact with Orai1 to form functional SOCs activated by STIM1 in a variety of cell types. In that model, STIM1 may open TRPC channels by electrostatic interaction through its polybasic lysine-rich domains (Cheng et al. 2013). Conversely, STIM1/Orai1 and TRPC channels have been shown to bind and inhibit voltage-gated L-type $\mathrm{Ca}^{2+}$ channels in some models (Moreno \& Vaca 2011, Sabourin et al. 2011).

Compared with the advances in many cell types, the physiological function of TRPC/Orai1-dependent SOCE is very unclear in $\beta$-cells.

\section{SOCE in insulin secretion: from capacitative $\mathrm{Ca}^{2+}$ entry to the role of the STIM-Orai-TRPC complex}

As mentioned in the introduction, pancreatic $\beta$-cells are excitable cells that depolarize in response to high glucose concentration. This electrical activity is characterized by typical bursting impulses, which in turn lead to $\left[\mathrm{Ca}^{2+}\right]_{\mathrm{i}}$ oscillations and subsequent insulin release oscillations. Thus, pancreatic $\beta$-cells rely largely on electrical activity and VDCC for extracellular $\mathrm{Ca}^{2+}$ influx. Nevertheless, it has been known for a long time that during glucoseinduced depolarization, the increase in $\left[\mathrm{Ca}^{2+}\right]_{\mathrm{i}}$ is mediated not only by $\mathrm{Ca}^{2+}$ influx through VDCC, but also by $\mathrm{Ca}^{2+}$

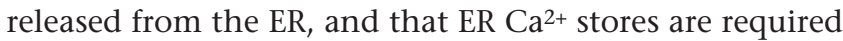
to maintain the amplitude of glucose-induced $\left[\mathrm{Ca}^{2+}\right]_{\mathrm{i}}$ oscillations over time. $\mathrm{ER} \mathrm{Ca}^{2+}$ release occurs both through passive leakage and active receptor-mediated release. Of note, in $\beta$-cells, active ER $\mathrm{Ca}^{2+}$ release is mainly due to $\mathrm{IP}_{3}$-induced $\mathrm{Ca}^{2+}$ release. $\mathrm{Ca}^{2+}$-induced $\mathrm{Ca}^{2+}$ release (CICR)

Published by Bioscientifica Ltd. 
through RyRs may also occur upon large VDCC-mediated $\mathrm{Ca}^{2+}$ influx. However, this process is still controversial (reviewed in Gilon et al. 2014).

Glucose-induced $\mathrm{Ca}^{2+}$ influx through VDCC contributes to refilling ER $\mathrm{Ca}^{2+}$ stores. However, $\mathrm{Ca}^{2+}$ efflux also occurs and VDCC alone cannot ensure ER $\mathrm{Ca}^{2+}$ homeostasis at all times. This is especially true in the case of stimulation with $\mathrm{IP}_{3}$-producing agents such as acetylcholine (ACh), which triggers massive ER $\mathrm{Ca}^{2+}$ export (reviewed in Gilon \& Henquin 2001). Early studies performed in hyperpolarized $\beta$-cells clearly demonstrated the presence of SOCE stimulated by $\mathrm{ER} \mathrm{Ca}^{2+}$ depletion in murine $\beta$-cells (Worley et al. 1994, Miura et al. 1996, Mears \& Zimliki 2004). This capacitative $\mathrm{Ca}^{2+}$ entry is of much lower amplitude than that induced by glucose through VDCC. However, it is important to keep in mind that $\left[\mathrm{Ca}^{2+}\right]_{\mathrm{i}}$ measurements in hyperpolarized cells mask a potential interplay with VDCC, thereby probably underestimating the influence of SOCs on $\mathrm{Ca}^{2+}$ influx.

Until recently, the identity of the channels mediating SOCE in $\beta$-cells remained unclear. Studies indicated that murine $\beta$-cells express several members of the TRPC protein family (reviewed in Jacobson \& Philipson 2007). STIM1 and Orai1 are also present, and ER $\mathrm{Ca}^{2+}$ depletion stimulates STIM1 translocation to the plasma membrane to co-cluster with Orai1 in murine $\beta$-cells (Tamarina et al.
2008, Tian et al. 2012). Using pharmacological approach and dominant negative mutants of STIM1, Orai1 and TRPC1, we demonstrated that STIM1 is instrumental in the activation of both Orai1 and TRPC1 channels upon store depletion, either in response to chemical ER depletion using thapsigargin or in response to the glucose-stimulated insulin secretion (GSIS) secretagogue ACh. We further demonstrated that TRPC1, Orai1 and STIM1 form a ternary complex upon ER $\mathrm{Ca}^{2+}$ depletion (Sabourin et al. 2015). The inhibition of these channels severely impairs the second phase of insulin release and fully blocks the potentiating effect of ACh on insulin secretion. Interestingly, blockade of either Orai1 or TRPC1 reduces the amplitude of the $\left[\mathrm{Ca}^{2+}\right]_{\mathrm{i}}$ oscillations in rat $\beta$-cells (Fig. 1), which supports a role of these proteins in the $\left[\mathrm{Ca}^{2+}\right]_{\mathrm{i}}$ dynamics of GSIS. In addition to TRPC1, we and others observed that rodent $\beta$-cells express TRPC3, -C4, -C5 and -C6 (Jacobson \& Philipson 2007, Sabourin et al. 2015). Our experiments showed that TRPC3 does not contribute to GSIS (Sabourin et al. 2015). However, it was recently suggested that TRPC3 may be involved in glucagon-like peptide 1 (GLP-1)-mediated $\mathrm{Ca}^{2+}$ influx in rat islets (Yamada et al. 2016). Further studies are required to determine the exact role of the different TRPC isoforms in $\beta$-cell function in response to glucose and secretagogues, as well as their potential interactions with VDCC.

A

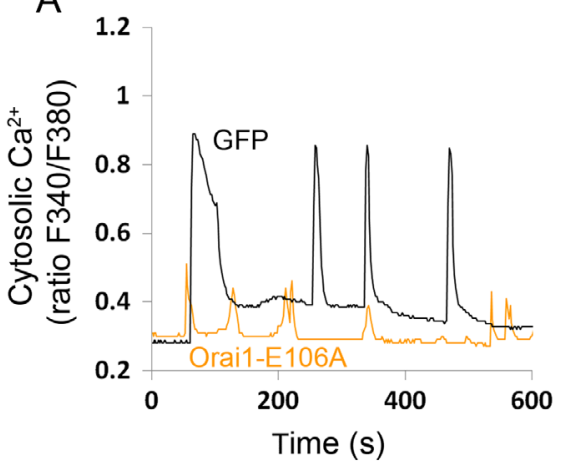

B
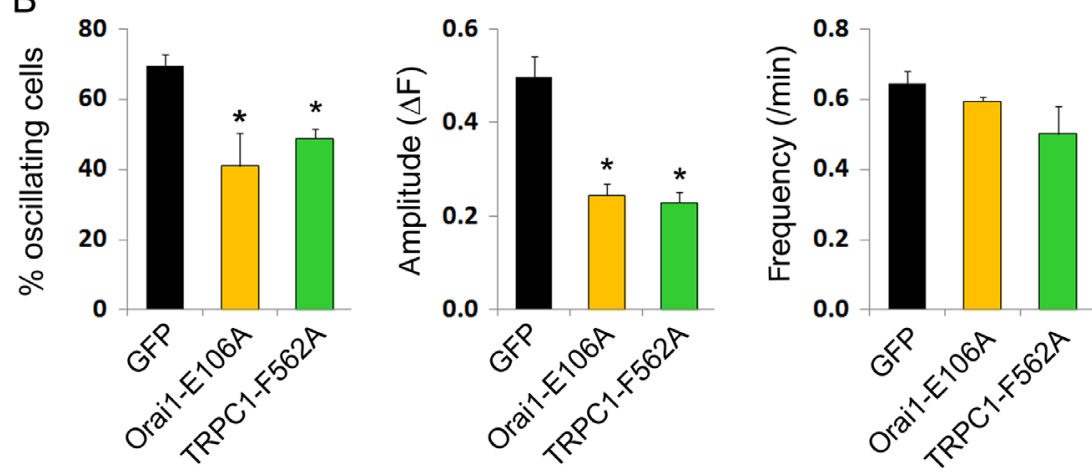

Figure 1

Orai1 and TRPC1 blockade reduces the number of oscillating cells and the amplitude of glucoseinduced intracellular $\mathrm{Ca}^{2+}$ oscillations in the rat insulinoma cell line INS-1E. Fura-2/AM imaging in INS-1E cells transfected with dominant negative Orai1-E106A or TRPC1-F562A mutants in KRBH supplemented with $20 \mathrm{mM}$ glucose and $2 \mathrm{mM}$ $\mathrm{Ca}^{2+}$. Transfected cells were loaded with $3 \mu \mathrm{M}$ Fura-2/AM plus 10\% (w/v) Pluronic F-127 (Life Technologies) for $45 \mathrm{~min}$ in $\mathrm{KRBH}$ supplemented with $2 \mathrm{mM}$ glucose. Cells were acquired after Fura-2/AM excitation at 340/380 nm with a Visichrome holographic monochromator. Images were treated with MetaFluor to evaluate the ratio of fluorescence emitted at 340 and $380 \mathrm{~nm}$ and the fluorometric signal was measured as the $\Delta \mathrm{F}$ (340/380 $\mathrm{nm}$ ratio). (A) Representative traces of INS-1E cells transfected with the control GFP, Orai1-E106A or TRPC1-F562A mutants. (B) Quantitative assessment of the percentage of transfected cells oscillating, of the $\mathrm{Ca}^{2+}$ oscillations amplitude $(\Delta \mathrm{F})$, and oscillations frequency per $\min . n=4$ experiments, $n=15$ investigated cells/experiment minimum. 
Early studies clearly demonstrated the existence of an inward current triggered by ER $\mathrm{Ca}^{2+}$ depletion in murine $\beta$-cells, which could depolarize the plasma membrane and activate VDCC (Worley et al. 1994, Dyachok \& Gylfe 2001, Mears \& Zimliki 2004). According to this hypothesis, the ER depletion and SOCs opening strongly amplify glucoseinduced depolarization in murine $\beta$-cells (Miura et al. 1996, Roe et al. 1998). Of note, the TRPC3 and -C6 channels have been shown, in vascular smooth muscle cells, to trigger sufficient depolarization to open VDCC (Soboloff et al. 2005, Wang et al. 2008). Careful electrophysiology studies should be undertaken to determine the impact of the ternary complex Orai1/TRPC1/STIM1 in the electrical activity of $\beta$-cells.

In contrast, reports indicate that STIM1/Orai1 and TRPC channels may suppress VDCC via physical interaction in various cell types (Moreno \& Vaca 2011, Sabourin et al. 2011). The direct or functional interactions between SOCs and VDCC in $\beta$-cells deserve further investigations to elucidate the effects of SOCE on VDCC and resulting regulation of GSIS.

Aberrant SOCE is observed in a growing number of diseases, such as cardiovascular, pulmonary, skin diseases and inflammation. Also, mutations in TRPC proteins have been linked to several channelopathies, and single-nucleotide polymorphisms in the TRPC1 gene are associated with type 2 diabetes mellitus (T2DM) (reviewed in Nilius \& Szallasi 2014). Mutations in STIM1 and ORAI1 have also been linked to devastating immunodeficiency diseases termed CRAC channelopathies. Additionally, STIM1/Orai1 dysregulations are involved in a broad range of diseases including cancer, neurodegenerative and cardiovascular diseases (Prakriya 2013). In our recent study, we demonstrated that SOCE function is altered in a rat $\beta$-cell line after prolonged exposure to high concentration of glucose (Sabourin et al. 2015), suggesting a potential dysfunction of SOCE in diabetes. So far, no link was found between Orai1, STIM1 or TRPCs and $\beta$-cell defects. Unfortunately, no data are available yet reporting the role of these proteins in insulin secretion in vivo. The study of mouse invalidated for Orai1 and STIM1 has demonstrated the importance of these proteins in development and growth (reviewed in Feske 2009), but their crippling phenotypes do not allow for proper study of $\beta$-cell function (Orai1-/- mice on a mixed genetic background are smaller, immunodeficient and present alopecia; Stim $1^{-/}$mice generated in various ways perish in utero or perinatali of respiratory failure). The limited current knowledge for this potentially key mechanism in insulin release calls for the generation of tissue-specific knockout mice to evaluate the importance of SOCE in vivo in normal and pathological situations leading to diabetes.

\section{Other TRP channels in $\beta$-cells}

Other reports point out to additional channels of the TRP family as key players in $\beta$-cell function. Rodent and human primary $\beta$-cells and/or insulin-secreting cell lines express a variety of TRP channels belonging to the vanilloid (TRPV2 and -4), ankyrin (TRPA1) and melastatin (TRPM2-5) subfamilies (reviewed in Colsoul et al. 2013).

TRPM2 forms functional $\mathrm{Ca}^{2+}$-permeable channels in $\beta$-cells, which contribute to insulin release in response to glucose and exendin-4 (a GLP-1 receptor agonist) and Trpm2-/- mice show hypoinsulinemia and resultant hyperglycemia. However, TRPM2 seems to regulate insulin secretion independently of its role as a $\mathrm{Ca}^{2+}$ channel and no correlation was detected between TRPM 2 variants and the risk for developing T2DM. GSIS is also significantly impaired in pancreatic islets from Trpm5-/- mice, resulting in impaired glucose tolerance. This appears to be due to a lack of glucose-induced fast $\left[\mathrm{Ca}^{2+}\right]_{\mathrm{i}}$ oscillations in those mice. In their study, Colsoul and coworkers hypothesized that these oscillations are lost due to an impairment of the $\mathrm{Ca}^{2+}$-activated $\mathrm{Na}^{+} / \mathrm{K}^{+}$current, which could be driven by the monocation-permeable TRPM5 channels. New reports further suggest a role of TRPM5 in the potentiating effect of GLP-1 in rat islets (Krishnan et al. 2014) and the GLP-1 effects are negligible in Trpm $4^{-/-}$or Trpm5 $5^{-/-}$islets (Shigeto et al. 2015). Of note, an association was recently found between TRPM5 variants and prediabetic phenotypes in individuals who are at risk for developing T2DM. The $\mathrm{Ca}^{2+}$ activated TRPM3 channels are also present in rodent and human islets. However, despite some findings indicating that these channels might form functional channels in $\beta$-cells, it is unlikely that they play a major role in physiologic insulin release. Finally, TRPA1 activation by mustard oil and 4-hydroxy-2-nonenal stimulates insulin release and the TRPA1 antagonist HC-030031 inhibits GSIS, suggesting a role for TRPA1 in $\beta$-cell function (Colsoul et al. 2013).

\section{Conclusion}

The model suggesting that GSIS relies only on VDCC has shown its limits and accumulating evidence now supports a growing importance for voltage-independent cation channels in $\beta$-cell function. These channels offer a new paradigm of $\left[\mathrm{Ca}^{2+}\right]_{\mathrm{i}}$ dynamics and pancreatic $\beta$-cell

Published by Bioscientifica Ltd 
excitability. Our findings especially stress the critical role of the ternary TRPC1/Orai1/STIM1 complex in $\mathrm{Ca}^{2+}$ influx, the amplitude of $\mathrm{Ca}^{2+}$ oscillations, and correlated GSIS. The existence of Orai2 and -3 , as well as STIM2, and other TRP proteins potentially interacting with the Orai1/TRPC1/STIM1 complex, calls for further studies to characterize the regulation of this new class of channels in $\beta$-cells.

\section{Declaration of interest}

The authors declare that there is no conflict of interest that could be perceived as prejudicing the impartiality of this review.

\section{Funding}

This work was supported by the Swiss National Science Foundation (grant number 31003A-155897 to F Allagnat) and by Agence Nationale de la Recherche (grant number ANR-15-CE14-0005 to J Sabourin).

\section{Acknowledgements}

We thank Professors Eric Raddatz and Jacques-Antoine Haefliger for their support. We thank Professor Shmuel Muallem (University of Texas Southwestern Medical Center, Dallas, TX, USA), who kindly provided the mutant TRPC1 (F562A) construct, and Professor Michael D Cahalan (University of California, Irvine, CA, USA), who kindly provided the mutant Orai (E106D).

\section{References}

Cheng KT, Ong HL, Liu X \& Ambudkar IS 2013 Contribution and regulation of TRPC channels in store-operated Ca2+ entry. Current Topics in Membranes 71 149-179. (doi:10.1016/b978-0-12-4078703.00007-x)

Colsoul B, Nilius B \& Vennekens R 2013 Transient receptor potential (TRP) cation channels in diabetes. Current Topics in Medicinal Chemistry 13 258-269. (doi:10.2174/1568026611313030004)

Dyachok O \& Gylfe E 2001 Store-operated influx of $\mathrm{Ca}(2+)$ in pancreatic beta-cells exhibits graded dependence on the filling of the endoplasmic reticulum. Journal of Cell Science 114 2179-2186.

Feske S 2009 ORAI1 and STIM1 deficiency in human and mice: roles of store-operated $\mathrm{Ca} 2+$ entry in the immune system and beyond. Immunological Reviews 231 189-209. (doi:10.1111/j.1600-065X. 2009.00818.x)

Gilon P \& Henquin JC 2001 Mechanisms and physiological significance of the cholinergic control of pancreatic beta-cell function. Endocrine Reviews 22 565-604. (doi:10.1210/er.22.5.565)

Gilon P, Chae HY, Rutter GA \& Ravier MA 2014 Calcium signaling in pancreatic beta-cells in health and in Type 2 diabetes. Cell Calcium 56 340-361. (doi:10.1016/j.ceca.2014.09.001)

Jacobson DA \& Philipson LH 2007 TRP channels of the pancreatic beta cell. Handbook of Experimental Pharmacology 179 409-424. (doi:10.1007/978-3-540-34891-7_24)

Krishnan K, Ma Z, Bjorklund A \& Islam MS 2014 Role of transient receptor potential melastatin-like subtype 5 channel in insulin secretion from rat beta-cells. Pancreas 43 597-604. (doi:10.1097/ MPA.0000000000000027)

Mears D \& Zimliki CL 2004 Muscarinic agonists activate Ca2+ store-operated and -independent ionic currents in insulin-secreting HIT-T15 cells and mouse pancreatic beta-cells. Journal of Membrane Biology 197 59-70. (doi:10.1007/s00232-003-0642-y)

Miura Y, Gilon P \& Henquin JC 1996 Muscarinic stimulation increases $\mathrm{Na}+$ entry in pancreatic B-cells by a mechanism other than the emptying of intracellular Ca2+ pools. Biochemical and Biophysical Research Communications 224 67-73. (doi:10.1006/bbrc.1996.0985)

Moreno C \& Vaca L 2011 SOC and now also SIC: store-operated and storeinhibited channels. IUBMB Life 63 856-863. (doi:10.1002/iub.547)

Nilius B \& Szallasi A 2014 Transient receptor potential channels as drug targets: from the science of basic research to the art of medicine. Pharmacological Reviews 66 676-814. (doi:10.1124/pr.113.008268)

Prakriya M 2013 Store-operated Orai channels: structure and function. Current Topics in Membranes 71 1-32. (doi:10.1016/b978-0-12407870-3.00001-9)

Putney JW Jr 1986 A model for receptor-regulated calcium entry. Cell Calcium 7 1-12. (doi:10.1016/0143-4160(86)90026-6)

Roe MW, Worley JF 3rd, Qian F, Tamarina N, Mittal AA, Dralyuk F, Blair NT, Mertz RJ, Philipson LH \& Dukes ID 1998 Characterization of a $\mathrm{Ca} 2+$ release-activated nonselective cation current regulating membrane potential and [Ca2+]i oscillations in transgenically derived beta-cells. Journal of Biological Chemistry 273 10402-10410. (doi:10.1074/jbc.273.17.10402)

Sabourin J, Robin E \& Raddatz E 2011 A key role of TRPC channels in the regulation of electromechanical activity of the developing heart Cardiovascular Research 92 226-236. (doi:10.1093/cvr/cvr167)

Sabourin J, Le Gal L, Saurwein L, Haefliger JA, Raddatz E \& Allagnat F 2015 Store-operated Ca2+ entry mediated by Orai1 and TRPC1 participates to insulin secretion in rat beta-cells. Journal of Biological Chemistry 290 30530-30539. (doi:10.1074/jbc.m115.682583)

Shigeto M, Ramracheya R, Tarasov AI, Cha CY, Chibalina MV, Hastoy B, Philippaert K, Reinbothe T, Rorsman N, Salehi A, et al. 2015 GLP-1 stimulates insulin secretion by PKC-dependent TRPM4 and TRPM5 activation. Journal of Clinical Investigation 125 4714-4728. (doi:10.1172/JCI81975)

Soboloff J, Spassova M, Xu W, He LP, Cuesta N \& Gill DL 2005 Role of endogenous TRPC6 channels in $\mathrm{Ca} 2+$ signal generation in A7r5 smooth muscle cells. Journal of Biological Chemistry $\mathbf{2 8 0}$ 39786-39794. (doi:10.1074/jbc.M506064200)

Tamarina NA, Kuznetsov A \& Philipson LH 2008 Reversible translocation of EYFP-tagged STIM1 is coupled to calcium influx in insulin secreting beta-cells. Cell Calcium 44 533-544. (doi:10.1016/j. ceca.2008.03.007)

Tian G, Tepikin AV, Tengholm A \& Gylfe E 2012 cAMP induces stromal interaction molecule 1 (STIM1) puncta but neither Orai1 protein clustering nor store-operated Ca2+ entry (SOCE) in islet cells. Journal of Biological Chemistry 287 9862-9872. (doi:10.1074/jbc.M111.292854)

Wang Y, Deng X, Hewavitharana T, Soboloff J \& Gill DL 2008 Stim, ORAI and TRPC channels in the control of calcium entry signals in smooth muscle. Clinical and Experimental Pharmacology and Physiology 35 1127-1133. (doi:10.1111/j.1440-1681.2008.05018.x)

Worley JF 3rd, McIntyre MS, Spencer B \& Dukes ID 1994 Depletion of intracellular $\mathrm{Ca} 2+$ stores activates a maitotoxin-sensitive nonselective cationic current in beta-cells. Journal of Biological Chemistry 269 32055-32058.

Yamada H, Yoshida M, Ito K, Dezaki K, Yada T, Ishikawa SE \& Kakei M 2016 Potentiation of glucose-stimulated insulin secretion by the GPR40-PLC-TRPC pathway in pancreatic beta-cells. Scientific Reports 6 25912. (doi:10.2165/00024677-200403030-00002)

Received in final form 8 July 2016

Accepted 2 September 2016

Accepted Preprint published online 2 September 2016

Published by Bioscientifica Ltc. http://jme.endocrinology-journals.org

DOI: 10.1530/JME-16-0106
() 2016 Society for Endocrinology Printed in Great Britain 